\title{
Abbauplanung der Schwebenrückgewinnung im Gipsbergbau Preinsfeld
}

\author{
Angelika Fischer ${ }^{1}$, Philipp Hartlieb ${ }^{1}$ und Johannes Daul ${ }^{2}$
}

'Lehrstuhl für Bergbaukunde, Bergtechnik und Bergwirtschaft, Department Mineral Resources Engineering, Montanuniversität Leoben, Leoben, Österreich ${ }^{2}$ Lafarge Zementwerke $\mathrm{GmbH}$, Wien, Österreich

Online publiziert 16. April 2020

Zusammenfassung: Im Gipsbergbau Preinsfeld in Niederösterreich wurde bis 2001 untertägig Gips gewonnen. Durch die Abbaumethode des Örter-Festenbaus auf Teilsohlen sind Schweben mit einer Mächtigkeit von circa $4 \mathrm{~m}$ zurückgeblieben. Im Rahmen einer Diplomarbeit wurde die noch vorhandene Menge Gips analysiert und eine geeignete Abbaumethode für die Schwebenrückgewinnung ausgewählt. Die Vortriebsmethode Bohren und Sprengen wurde dem maschinellen Vortrieb mittels Teilschnittmaschine gegenübergestellt. Aufgrund technischer und wirtschaftlicher Aspekte wurde die Teilschnittmaschine als geeigneter angesehen und die Materialförderung darauf abgestimmt. Mit Hilfe der Geologie- und Bergbauplanungssoftware Geovia Surpac ${ }^{\mathrm{TM}}$ wurde ein dreidimensionales Bergbaumodell für unterschiedliche Abbaumethoden erstellt. Die Abbaumethode des klassischen Örter-Festenbaus wurde mit verschiedenen Streckenprofilen geplant und einem Kammerbau mit Vollversatz gegenübergestellt. Die Entscheidung der Abbaumethode fiel unter Berücksichtigung von wirtschaftlichen, technischen und sicherheitsrelevanten Aspekten zugunsten des Kammerbaus aus. Dieser wurde in Folge im Detail unter anderem hinsichtlich Sequenzierung, Bewetterung, Kosten und jährlicher Produktion geplant.

Schlüsselwörter: Untertagebergbau, Kammerbau, Gips, Preinsfeld, Surpac ${ }^{\mathrm{TM}}$, Teilschnittmaschine, Vollversatz

Development of an Extraction Method for the Sill Pillars in the Gypsum Mine Preinsfeld

Abstract: Until 2001, gypsum was extracted in an underground operation from the gypsum mine Preinsfeld, Aus-

\footnotetext{
Dipl.-Ing. Dr. P. Hartlieb ( $\triangle)$

Lehrstuhl für Bergbaukunde, Bergtechnik und Bergwirtschaft,

Department Mineral Resources Engineering,

Montanuniversität Leoben,

Franz Josef Str. 18,

8700 Leoben, Österreich

Philipp.hartlieb@unileoben.ac.at
}

tria. The initially used mining method was room and pillar mining. Due to the former extraction on sublevels, there are still pillars of approximately $4 \mathrm{~m}$ height left to mine. Within the scope of a master thesis, the remaining amount of gypsum in the mine was evaluated and an appropriate mining method to extract it was developed.

The extraction method of blasting was compared to machine excavation with a roadheader. After the decision was taken in favor of the roadheader, the hauling fleet was adjusted to it.

By using the geology and mine planning software Geovia Surpac $^{\mathrm{TM}}$, a three-dimensional model of the mine was created for different mining methods. A classical room and pillar mining method was planned for different tunnel profiles and then compared to an adjusted cut and fill stoping method with backfill. After consideration of technical, economical and safety factors, the decision was made in favor of the cut and fill mining method. Therefore, a detailed study on the mine planning, mine ventilation, costs and life of mine was conducted.

Keywords: Underground mining, Cut and fill stoping, Gypsum, Preinsfeld, Surpac ${ }^{\top M}$, Roadheader, Backfill

\section{Einleitung}

Die Anfänge des Gipsbergbaues Preinsfeld lassen sich zumindest bis in das 17. Jahrhundert zurückverfolgen. Über die Jahre wurde der Gips sowohl untertägig als auch tagebaumäßig von verschiedenen Pächtern abgebaut. Im Jahr 1865 wurde ein circa $350 \mathrm{~m}$ langer Stollen aufgefahren, welcher in das derzeitige Grubengebäude mündet und als „Preinsfelder Stollen“ bezeichnet wird. Im Verlauf der Geschichte kam es immer wieder zu Stillstandsperioden, bis im Jahr 1962 die Gipsbergbau Preinsfeld Ges.m.b.H Nfg. KG, welche seit 1981 Eigentum der Lafarge Zementwerke G.m.b.H. ist, den Gipsbergbau übernahm [1].

In Anbetracht der geologischen Gegebenheiten und immer größer werdender Überlagerung wurde der damalige 
Tagebau im Jahr 1965 nach Untertage verlegt. Anfänglich wurde der Gips durch Bohren und Sprengen hereingewonnen, bis schließlich 1972 eine Teilschnittmaschine die Gewinnung übernahm [2].

Seit 2001 findet kein Abbau mehr statt und das bestehende Grubengebäude wird seitdem zur Sicherung der Oberfläche und des Grubengebäudes selbst verfüllt.

\section{Analyse des derzeitigen Bergbaues}

Das derzeitige Grubengebäude dehnt sich über insgesamt 8 Teilsohlen aus, welche sich von $400 \mathrm{~m}$ Seehöhe bis zu einer Teufe von $320 \mathrm{~m}$ Seehöhe aufteilen.

Die Ausdehnung misst in Streichrichtung etwa $800 \mathrm{~m}$, quer zum Streichen spannt sich das Grubengebäude zwischen 40 und $160 \mathrm{~m}$ auf. Der Gips wurde in einem ÖrterFestenbau ohne Versatz abgebaut, welcher untertägig von oben nach unten feldwärts vorangetrieben wurde. Die oberen Sohlen wurden durch Bohren und Sprengen abgebaut, während die unteren Sohlen mit Hilfe einer Teilschnittmaschine abgebaut wurden. Dabei wurden Strecken mit einem Querschnitt von $8 \mathrm{~m}$ Breite und $4 \mathrm{~m}$ Höhe aufgefahren und Festen mit einer Grundfläche von circa $4 \times 4 \mathrm{~m}$ stehen gelassen [2].

Die Gipslagerstätte selbst erstreckt sich in NordwestSüdost-Richtung und fällt mit circa $30-40^{\circ}$ ein. Der Gipskörper wird immer wieder von Toneinlagerungen gestört und von den Werfener Schichten als Nebengebirge umgeben [2].

Das durch das Unternehmen iC consulenten Ziviltechniker GesmbH vermessene und digitalisierte Grubengebäude wurde im Rahmen der Diplomarbeit analysiert. Auf dieser Basis wurde die Abbauplanung durchgeführt und ein dreidimensionales Modell zur Schwebenrückgewinnung erstellt. Einige Bereiche des Grubengebäudes sind aufgrund von Pingenbildungen oder zu geringer Schwebenmächtigkeit nicht abbaubar. Diese Bereiche wurden identifiziert und in der Abbauplanung ausgeklammert. Die übrig gebliebenen, abbauwürdigen Schweben wurden genauer betrachtet und auf deren Grundlage eine Abbaumethode geplant.

\section{Wahl der Vortriebsmethode}

Bei der Wahl der Vortriebsmethode für den Gipsbergbau Preinsfeld muss grundsätzlich zwischen einem konventionellen Vortrieb durch Bohr- und Sprengarbeit und einem maschinellen Vortrieb unterschieden werden. In der Vergangenheit wurden beide Vortriebsarten im Bergbau eingesetzt. Auf den oberen Sohlen wurde der Gips hauptsächlich durch Bohren und Sprengen gewonnen, während auf den unteren Sohlen eine Teilschnittmaschine zum Einsatz gekommen ist.

Unter Berücksichtigung der vorliegenden Lagerstätte, der Gebirgseigenschaften sowie der notwendigen Arbeitsschritte der beiden Vortriebsmethoden fällt die Entscheidung auf die Teilschnittmaschine. Die mit der Teilschnittmaschine einhergehenden Vorteile liegen in den geringeren
Anforderungen in Bezug auf die Sicherheit, Logistik und Bewetterung, im geringeren Personal- und Betriebsmittelaufwand sowie in der gleichmäßigeren Korngrößenverteilung des Hauwerkes. Nachteilig sind hingegen die höheren Investitionskosten sowie der höhere Wartungs- bzw. Instandhaltungsaufwand. Des Weiteren steht die gesamte Produktion still, wenn es zu einem unerwarteten Stillstand der Teilschnittmaschine kommen sollte. Eine kontinuierliche Wartung und gut geplanter Instandhaltungsplan sind demnach unerlässlich.

In der Vergangenheit war eine Teilschnittmaschine des Typs AM 50 der Voest-Alpine in Verwendung. Da diese Teilschnittmaschine in der Form nicht mehr produziert wird, wurde nach möglichen Alternativen gesucht, die eine kompakte Bauweise aufweisen, um auch in Bereichen mit Schwebenmächtigkeiten $<4 \mathrm{~m}$ gut operieren $\mathrm{zu}$ können. Gewählt wurde eine Teilschnittmaschine mit Querschneidkopf, von dem der gelöste Gips über Ladesterne und Kettenförderer direkt auf den bereitstehenden Untertagemuldenkipper geladen wird. Um Gipsanbackungen und den damit einhergehenden Meißelverschleiß gering halten zu können, muss auf eine genaue Reinigung des Schneidkopfes je Schicht geachtet werden.

\section{Auswahl des Abbauverfahrens}

Die Wahl einer geeigneten Abbaumethode wurde unter Berücksichtigung von Faktoren wie der Lagerstättengeometrie und Geologie, gewünschter Produktionsrate und Maschinenkapazität, den Kapital- und Betriebskosten, Sicherheitsfaktoren, Umwelteinflüssen sowie gebirgsmechanischen Faktoren getroffen [3].

Aufgrund der bisherigen Bergbautätigkeit und der daraus entstandenen Schweben, welche horizontal und mit einer Mächtigkeit von circa $4 \mathrm{~m}$ in der Lagerstätte liegen, kommen als Abbaumethoden vor allem der Örter-Festenbau und der Kammerbau in Frage. Bei beiden Methoden muss Versatz eingebracht werden, um die Tagesoberfläche zu schützen.

\section{1 Örter-Festenbau}

Im Rahmen der Masterarbeit wurden 3 Szenarien eines Örter-Festenbaus mit unterschiedlichen Geometrien geplant und in einem dreidimensionalen Surpac ${ }^{\mathrm{TM}}$ Modell dargestellt. Je nach gewählter Geometrie lassen sich bei dieser Abbaumethode Ausbringen von 62 bis $72 \%$ erzielen.

\subsection{Kammerbau}

Alternativ zu dem Örter-Festenbau wurde eine Art Kammerbau mit Primär- und Sekundärkammersequenz geplant. Ziel dieser Abbaumethode ist es, durch Einbringen von Versatz die Lagerstätte so vollständig wie möglich abzubauen. Um dies zu erreichen, werden von zwei parallel laufenden Hauptstrecken in Streichrichtung der Lagerstätte Kammern quer zum Streichen abgebaut und anschließend 
Abb. 1: Schematische Darstellung der Abbaukammern und Hauptstrecken

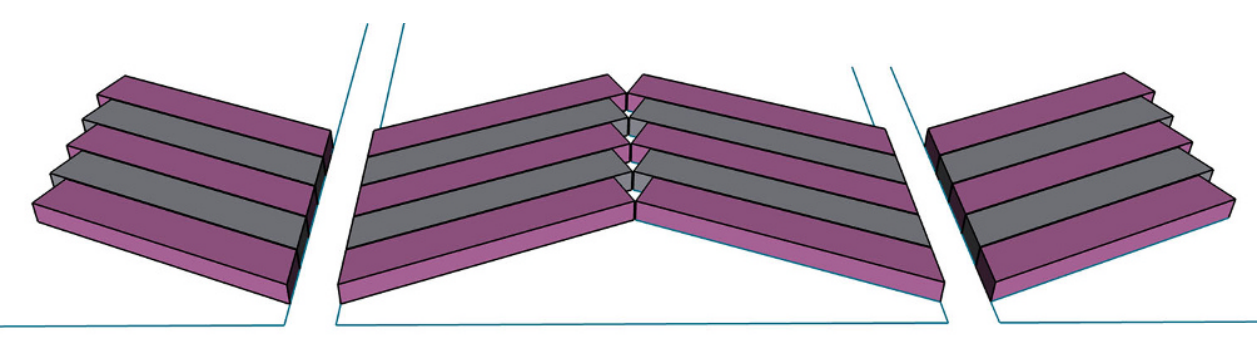

verfüllt. In einem Intervall von circa $50 \mathrm{~m}$ werden Querschläge aufgefahren, welche die beiden Hauptstrecken miteinander verbinden und zur Materialförderung bzw. Wetterführung dienen. Zwischen den einzelnen Kammern werden zunächst Pfeiler in den Dimensionen der Kammern stehengelassen und in einem zweiten Schritt abgebaut und ebenfalls verfüllt.

Die Kammern erstrecken sich auf eine Länge von ungefähr $45 \mathrm{~m}$ beidseits der Hauptstrecken. Um den Zugang zu den Kammern für die Teilschnittmaschine und die Muldenkipper zu erleichtern, werden die Kammern statt in einem $90^{\circ}$-Winkel in einem $60-65^{\circ}$-Winkel zu den Hauptstrecken aufgefahren. Dies steigert die Effektivität der Förderung und des Abbaus, da die Zeiten für das Wenden der Maschinen verringert werden können.

Die Hauptstrecken, wie auch die Abbaukammern, weisen einen Querschnitt von $8 \mathrm{~m}$ Breite und $4 \mathrm{~m}$ Höhe auf, sodass die Teilschnittmaschine den Vollquerschnitt in zwei Abbausequenzen bearbeiten kann. Abb. 1 zeigt eine schematische Darstellung der Abbaumethode. Durch Anwendung dieser Abbaumethode kann ein Ausbringen von circa $76 \%$ erreicht werden.

\subsection{Vergleich der Abbaumethoden}

Die verschiedenen Abbaumethoden wurden hinsichtlich technischer, wirtschaftlicher als auch sicherheitstechnischer Aspekte miteinander verglichen, um dadurch die am besten geeignete Abbaumethode wählen zu können. Dabei wurde entschieden, dass der Kammerbau die geeignetste Abbaumethode für den Abbau der Schweben des Gipsbergbau Preinsfeld darstellt. Das Ausbringen ist beim Kammerbau im Vergleich zu den anderen Optionen sehr hoch. Die Anforderungen an die Bewetterung und das Maschinenhandling sind geringer. Aus gebirgsmechanischer Sicht ist ebenfalls der Kammerbau vorzuziehen, da immer nur eine Abbaukammer offen steht und danach sofort verfüllt wird. Was den Versatz betrifft, so stellt der Kammerbau im Vergleich zu den anderen Optionen allerdings die höchsten Anforderungen. Entgegenwirkend ist das Einbringen des Versatzes in die Abbaukammern unkomplizierter, da in Summe weniger Barrikaden verbaut und Querwegigkeiten berücksichtigt werden müssen. Aufgrund dieser Entscheidungskriterien wurde der Kammerbau im weiteren Verlauf der Diplomarbeit im Detail geplant. Dazu wurden alle mit dem Bergbau zusammenhängenden Tätigkeiten detailliert betrachtet wie der Aufschluss der Schweben, die Sequenzierung, der Versatz, Förder- und Ladespiel, die
Bewetterung, die Abbauplanung und jährliche Produktion sowie die anfallenden Investitions- und Betriebskosten.

\section{Detailplanung Kammerbau mit Versatz}

Der Aufschluss der Lagerstätte erfolgt über eine Rampe durch den Preinsfelder Stollen, welcher auch in der Vergangenheit zum Einsatz gekommen ist, und taucht von dort dann in südlicher Richtung in die Lagerstätte ab. Über eine Kombination aus geraden Rampenstücken und Wendeln können die einzelnen Schweben erreicht werden. Um die Förderwege und Fluchtwege gering zu halten, liegt die Wendel circa in der Mitte der jeweiligen Schwebe und unterteilt die Schweben somit in ein Süd- und ein Nordfeld.

Die einzelnen Schweben wurden in der Detailplanung genau auf mögliche Gefahrenbereiche durch bestehende Pingen oder Bereiche mit zu geringer Mächtigkeit hin untersucht. Diese Bereiche wurden in der Abbauplanung weiters ausgeklammert.

Der allgemeine Abbau der Schweben führt von unten nach oben, beginnt also auf der untersten Schwebe und arbeitet sich in weiterer Folge bis zur obersten Sohle durch. Dadurch wird immer auf dem eingebrachten Versatz und nicht unter dem Versatz gearbeitet. Die Schweben selbst werden in ein Süd- und ein Nordfeld unterteilt. Die Abbaukammern und Hauptstrecken sind so ausgerichtet, dass die Kammern immer in Fahrtrichtung beidseits der Hauptstrecken aufgefächert sind. Zunächst wird das Südfeld vollständig abgebaut und verfüllt und anschließend aus Bewetterungsgründen und der Arbeitssicherheit verbarrikadiert.

Bei der Abbaumethode des Kammerbaus ist der Abbaufortschritt stark von der Art, dem Einbringen und den Aushärtezeiten und Festigkeiten des Versatzes abhängig. Mittels Rohrleitungen wird Pumpversatz in die verbarrikadierten Abbaukammern gepumpt. Erst wenn der Versatz in der abgebauten Kammer vollständig erhärtet ist, kann der Abbau einer daneben liegenden Abbaukammer vorgenommen werden.

Durch die gewählte Abbaumethode kann die Förderung des Gipses aus den Abbaukammern in einem Einbahnsystem geführt werden. (Abb. 2).

Die Bewetterung der Schweben erfolgt über die Wendel und einen Hauptwetterschacht, welcher bis zur untersten Schwebe reicht und einen zweiten kleineren Wetterschacht, welcher die Bewetterung der oberen Schweben sicherstellt. 
Abb. 2: Lade- und Förderspiel
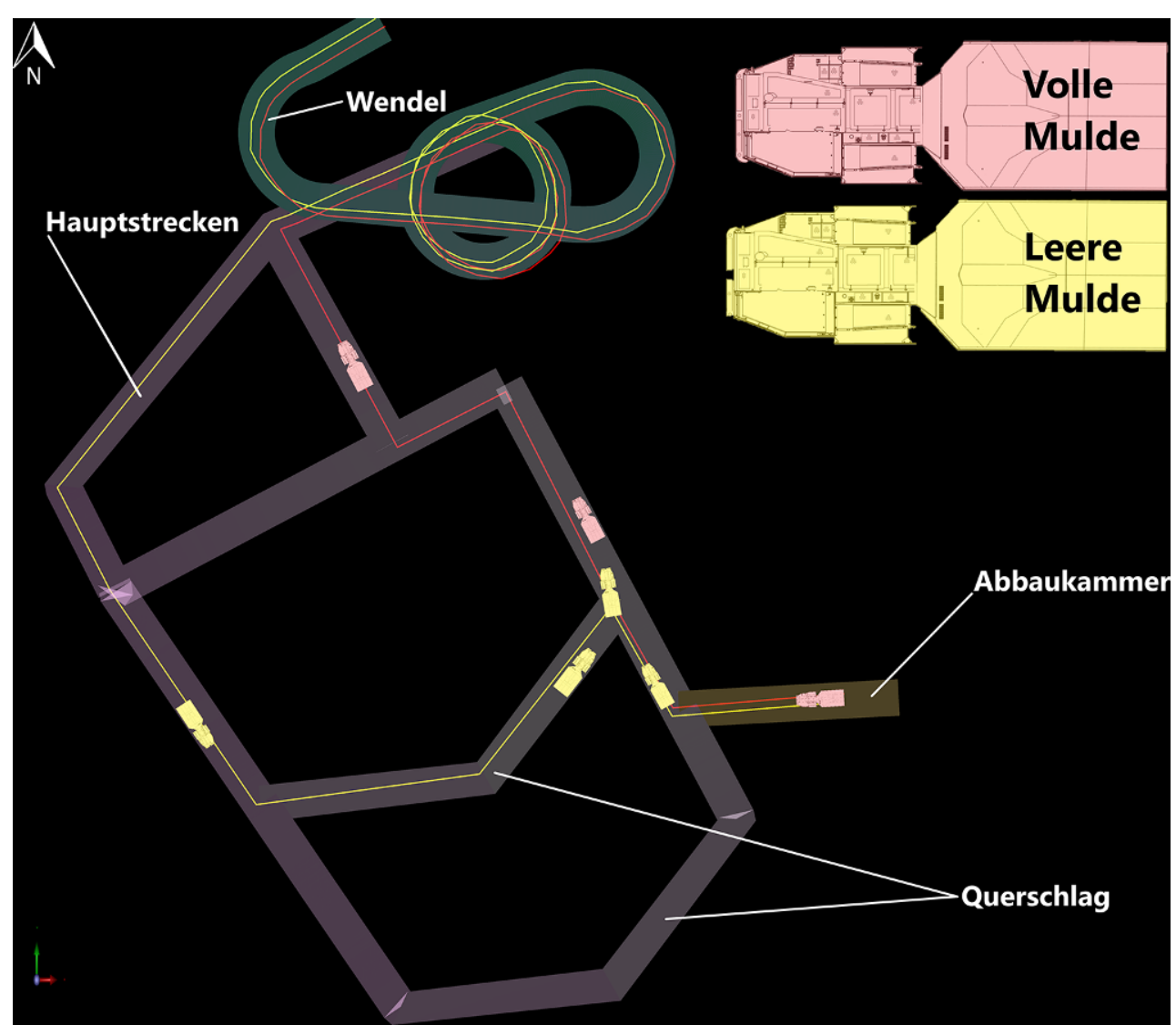

\section{Schlussfolgerung}

Die Gipslagerstätte Preinsfeld wurde in der Vergangenheit im Örter-Festenbau auf insgesamt 8 Teilsohlen abgebaut. Dabei wurden Schweben mit einer Mächtigkeit von circa $4 \mathrm{~m}$ zurückgelassen. Durch die Analyse des bestehenden Grubengebäudes wurden jene Schweben identifiziert, welche sich für einen Abbau eignen. Für das Lösen des Gipses wurde nach ausführlicher Analyse eine Teilschnittmaschine ausgewählt. Für die Abbauplanung wurde einerseits ein Örter-Festenbau mit unterschiedlichen Abbaugeometrien geplant und andererseits ein Kammerbau mit Vollversatz. Im direkten Vergleich der Abbaumethoden hat sich der Kammerbau als vorteilhaft herausgestellt. Vorteile liegen unter anderem in der einfacheren Bewetterung, einem effizienteren Maschinenhandling sowie dem höheren Ausbringen. Durch neue Technologien wie elektrisch angetriebene Muldenkipper und Ventilation on Demand können die Kosten und der Energiebedarf in Zukunft gering gehalten werden.

Funding. Open access funding provided by Montanuniversität Leoben.

Open Access Dieser Artikel wird unter der Creative Commons Namensnennung 4.0 International Lizenz veröffentlicht, welche die Nutzung, Vervielfältigung, Bearbeitung, Verbreitung und Wiedergabe in jeglichem Medium und Format erlaubt, sofern Sie den/die ursprünglichen Autor(en) und die Quelle ordnungsgemäß nennen, einen Link zur Creative Commons Lizenz beifügen und angeben, ob Änderungen vorgenommen wurden.
Die in diesem Artikel enthaltenen Bilder und sonstiges Drittmaterial unterliegen ebenfalls der genannten Creative Commons Lizenz, sofern sich aus der Abbildungslegende nichts anderes ergibt. Sofern das betreffende Material nicht unter der genannten Creative Commons Lizenz steht und die betreffende Handlung nicht nach gesetzlichen Vorschriften erlaubt ist, ist für die oben aufgeführten Weiterverwendungen des Materials die Einwilligung des jeweiligen Rechteinhabers einzuholen.

Weitere Details zur Lizenz entnehmen Sie bitte der Lizenzinformation auf http://creativecommons.org/licenses/by/4.0/deed.de.

\section{Literatur}

1. Holzer, H. F; Weber, F; Trimmel, F: Zur Geologie und Geophysik der Gipslagerstätte Preinsfeld bei Heiligenkreuz, N.-Ö., Berg- und Hüttenmännische Monatshefte 120 (1975), Nr. 12, S. 569-576

2. Daul, J.; Scheiber, M.; Bergmair, M.: Gips raus - Gips rein: Verfüllung des Gipsbergbaues Preinsfeld / NÖ, in: Meier, G. (Hrsg.): 9. Altbergbaukolloquium, Montanuniversität Leoben 2009, Essen: VGEVerlag, 2009, S. 278-284

3. Nelson, M. G.: Evaluation of Mining Methods and Systems, in: Darling, P. (ed.): SME Mining Engineering Handbook, 3rd ed., Englewood, CO: SME, 2011, S. 341-348

Hinweis des Verlags. Der Verlag bleibt in Hinblick auf geografische Zuordnungen und Gebietsbezeichnungen in veröffentlichten Karten und Institutsadressen neutral. 